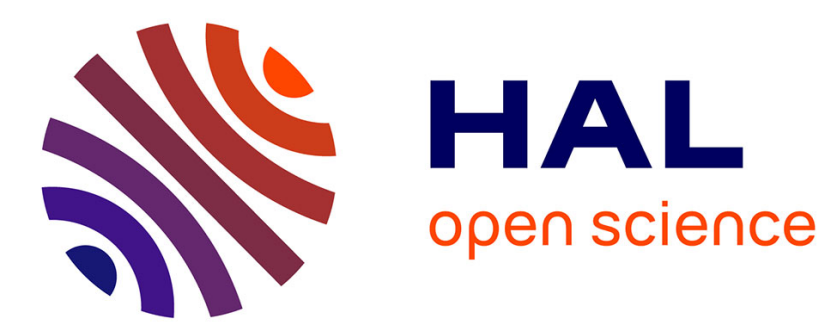

\title{
Shapes describing the alpha decay, cluster radioactivity, fusion, fission and fragmentation phenomena
}

\author{
Guy Royer, J. Jahan, N. Mokus
}

\section{To cite this version:}

Guy Royer, J. Jahan, N. Mokus. Shapes describing the alpha decay, cluster radioactivity, fusion, fission and fragmentation phenomena. Physica Scripta, 2018, 93, pp.094004. 10.1088/1402-4896/aad6fb . in2p3-01884469

\author{
HAL Id: in2p3-01884469 \\ https://hal.in2p3.fr/in2p3-01884469
}

Submitted on 1 Oct 2018

HAL is a multi-disciplinary open access archive for the deposit and dissemination of scientific research documents, whether they are published or not. The documents may come from teaching and research institutions in France or abroad, or from public or private research centers.
L'archive ouverte pluridisciplinaire $\mathbf{H A L}$, est destinée au dépôt et à la diffusion de documents scientifiques de niveau recherche, publiés ou non, émanant des établissements d'enseignement et de recherche français ou étrangers, des laboratoires publics ou privés. 


\title{
Shapes describing the alpha decay, cluster radioactivity, fusion, fission and fragmentation phenomena
}

\author{
G Royer, J Jahan and N Mokus \\ Laboratoire Subatech, UMR: IMT - IN2P3/CNRS -Université, Nantes 44, France
}

\begin{abstract}
To model the alpha decay, cluster radioactivity, fusion, fission and fragmentation phenomena of microscopic or macroscopic distributions of matter or charge it is useful to simulate these deformed physical objects by geometric shapes allowing the determination of their root mean square radius, volume, surface and Coulomb energies as well as their moments of inertia and quadrupole moments. Most of the shapes used in macroscopic nuclear physics are briefly recalled. In particular, several shape sequences that we have used previously, mainly formed from generalized lemniscatoids, are more extensively detailed. They allow to describe the transitions from one compact configuration to several ones or vice versa.
\end{abstract}

e-mail: royer@subatech.in2p3.fr

\section{Introduction}

In chemistry, mechanics, astrophysics, hydrodynamics, nuclear physics,... the transition from one almost spherical system towards several spherical or ellipsoidal objects or vice versa (alpha decay, cluster radioactivity, fragmentation, fission, fusion,...) may be described in regarding the evolution of the density profile in the system during the deformation or in following the deformation of specific assumed geometric shape sequences. In this last approach to determine the macroscopic energy it is necessary to know the main characteristics of the shape: the position of the centre of inertia, the root mean square radius, the volume, the surface, the Coulomb function, the moments of inertia, the quadrupole moment,...

An extensive review of the geometrical relationships used in macroscopic nuclear physics may be found in Ref. [1]. In a first part, most of these shape sequences used to describe the nuclear states and reactions are reviewed. In the following sections the definitions and geometric properties of different shape sequences that we have previously used and bring together recently [2] are recalled. Within macro-microscopic models they allow the study of the fusion [3, 4], fission [5, 6], fragmentation $[7,8]$ and alpha decay and cluster radioactivity processes $[9,10,11]$. The following shapes will be successively presented: one ellipsoid, two ellipsoids, elliptic and hyperbolic generalized lemniscatoids, prolate compact ternary shapes, bubbles and tori. 
To study $\mathrm{n} \alpha$-nuclei as nuclear molecules, different configurations such as linear chain, triangle, square, tetrahedron, pentagon, square bipyramid, trigonal pyramid, hexagon, octahedron, octagon and cube have also been selected [12].

\section{Mini-review of the main theoretical nuclear shapes}

Within macroscopic models such as the liquid drop model or droplet model the total energy of a system is, mainly, the sum of the volume, surface and Coulomb energy and shape-dependent functions are used. For charge or matter distributions of constant density the relative shape-dependent surface $B_{s}$ and Coulomb (or gravitational) $B_{c}$ functions are respectively [1]

$$
\begin{aligned}
B_{s} & =\int_{\sigma} \frac{d \sigma}{4 \pi R_{0}{ }^{2}}, \\
B_{c} & =\frac{15}{16 \pi^{2} R_{0}{ }^{5}} \int d \tau \int \frac{d \tau^{\prime}}{\left|\vec{r}-\vec{r}^{\prime}\right|},
\end{aligned}
$$

where $R_{0}$ is the radius of the sphere of same volume.

When the shapes are axially symmetric, $B_{c}$ reduces to

$$
B_{c}=0.5 \int \frac{v\left(\theta_{i}\right)}{v_{0}}\left[\frac{R\left(\theta_{i}\right)}{R_{0}}\right]^{3} \sin \left(\theta_{i}\right) d \theta_{i} .
$$

The potential $v(\theta)$ is determined at the surface of the shape, $v_{0}$ being the surface potential of the sphere

$\frac{v\left(\theta_{i}\right)}{v_{0}}=\frac{3}{2 \pi R_{0}^{2}} \int \frac{\rho\left[\left(\rho_{i}+\rho\right) \frac{d z}{d \theta}+\left(z_{i}-z\right) \frac{d \rho}{d \theta}\right] K(k)-\frac{1}{2}\left[\left(\rho_{i}+\rho\right)^{2}+\left(z_{i}-z\right)^{2}\right] \frac{d z}{d \theta} D(k)}{\sqrt{\left(\rho_{i}+\rho\right)^{2}+\left(z_{i}-z\right)^{2}}} d \theta(4)$

where $\rho=R(\theta) \sin \theta, z=R(\theta) \cos \theta . D(k)$ and $K(k)$ are calculated from elliptic integrals. These shape-dependent $B_{s}$ and $B_{c}$ functions are dimensionless and equal to one at the sphere.

For axially symmetric shapes the inverse effective moment of inertia is defined by

$$
I_{e f f}^{-1}=I_{\|}^{-1}-I_{\perp}^{-1} \text {. }
$$

$I_{\|}$and $I_{\perp}$ are the parallel and perpendicular moments of inertia.

The dimensionless quadrupole moment $Q$ relatively to the $\mathrm{z}$ axis is given by

$$
Q=\frac{1}{R_{0}^{5}} \iiint\left(3 z^{2}-r^{2}\right) d \tau
$$

The parameter $\beta$, often used in $\gamma$ spectroscopy, follows

$$
\begin{aligned}
& \beta=\frac{0.75}{\sqrt{5 \pi}} Q R_{0}^{2}\left\langle r^{2}\right\rangle^{-1}, \\
& \beta=2 \sqrt{\frac{\pi}{5}} \frac{I_{\perp}-I_{\|}}{I_{\perp}+0.5 I_{\|}} .
\end{aligned}
$$




\subsection{Small deformations}

For small oblate or prolate deformations from the sphere (nuclear ground states, planets) the spheroidal expansions have been used [13] within a small expansion of the eccentricity e. For prolate spheroids, the minor a and major c semi axes are given by

$$
a=R_{0}\left(1-\frac{1}{6} e^{2}-\frac{5}{72} e^{4}+\ldots\right), \quad c=R_{0}\left(1+\frac{1}{3} e^{2}+\frac{2}{9} e^{4}+\ldots\right) .
$$

For oblate spheroids $e^{2}$ must be replaced by $-e^{2}$. The shape-dependent functions are:

$$
\begin{aligned}
& I_{\perp}=1+\frac{1}{6} e^{2}+\frac{2}{9} e^{4}+\frac{35}{162} e^{6} \ldots, \quad I_{\|}=1-\frac{1}{3} e^{2}-\frac{1}{9} e^{4}-\frac{5}{81} e^{6} \ldots, \\
& I_{e f f}=\frac{1}{2} e^{2}+\frac{5}{12} e^{4}+\frac{85}{216} e^{6} \ldots, \\
& Q=\frac{8 \pi}{15} e^{2}\left(1+\frac{2}{3} e^{2}+\frac{5}{9} e^{4}+\frac{40}{81} e^{6} \ldots\right), \\
& B_{s}=1+\frac{2}{45} e^{4}+\frac{116}{2835} e^{6} \ldots, \quad B_{c}=1-\frac{1}{45} e^{4}-\frac{64}{2835} e^{6} \ldots, \\
& r_{r m s}^{2}=\frac{3}{5}\left(1-\frac{1}{9} e^{2}+\frac{1}{9} e^{4}+\frac{10}{81} e^{6} \ldots\right) .
\end{aligned}
$$

Several harmonic expansions have been proposed [1].

The $\alpha_{n}$ parameterization is defined by $[14,15,16]$

$$
R(\theta)=\lambda^{-1} R_{0}\left[1+\sum_{n=1}^{N} \alpha_{n} P_{n}(\cos \theta)\right],
$$

$\mathrm{N}$ being a cutoff parameter, $\lambda$ a parameter allowing the volume conservation and $P_{n}$ a Legendre polynomial.

The volume conservation can be included into the deformation parameters [15]

$$
R(\theta)=R_{0}\left[1+\sum_{n=0}^{N} a_{n} P_{n}(\cos \theta)\right] .
$$

The $\beta_{n}$ parameterization [17] takes into account the volume conservation only up to quadratic terms in the deformation parameters

$$
R(\theta)=R_{0}\left[1+\sum_{n=2}^{N}\left(\beta_{n} Y_{n 0}(\theta)-\frac{\beta_{n}^{2}}{4 \pi}\right)\right] .
$$

$Y_{n 0}$ are the spherical harmonics. The parameter $\beta_{2}$ is usually denoted by $\beta$.

The relative quadrupole and hexadecapole moments read [18]

$$
\begin{aligned}
& Q=4 \sqrt{\pi / 5}\left(\beta_{2}+0.36 \beta_{2}^{2}+0.967 \beta_{2} \beta_{4}+0.328 \beta_{4}^{2}+0.023 \beta_{2}^{3}+\ldots\right) \\
& Q_{4}=\frac{4}{3} \sqrt{\pi}\left(\beta_{4}+0.725 \beta_{2}^{2}+0.983 \beta_{2} \beta_{4}+0.411 \beta_{4}^{2}+0.416 \beta_{2}^{3}+\ldots\right) .
\end{aligned}
$$

The $\varepsilon_{n}$ parameterization has also been defined to describe deformations greater than spheroidal deformations using expansions about a spheroid [19]. 
To define the semi-axes a, b and c of triaxial ellipsoids the parameters $\beta_{H}$ and $\gamma_{H}$ have been introduced by Hill and Wheeler [14],

$$
\begin{aligned}
& a=R_{0} \exp \left[\alpha_{H} \cos \left(\gamma_{H}-\frac{2 \pi}{3}\right)\right], \\
& b=R_{0} \exp \left[\alpha_{H} \cos \left(\gamma_{H}+\frac{2 \pi}{3}\right)\right], \\
& c=R_{0} \exp \left[\alpha_{H} \cos \left(\gamma_{H}\right)\right] .
\end{aligned}
$$

$0 \leq \gamma_{H}<\pi / 3$ and $\alpha$ is unrestricted.

$$
\beta_{H}=\sqrt{\frac{4 \pi}{5}} \alpha_{H}
$$

When $\gamma$ varies from 0 to $\pi / 3$ the ellipsoid changes from axially prolate to axially oblate shapes. The dimensionless surface and Coulomb functions are given by

$$
B_{s}=1+\frac{2}{5} \alpha_{H}^{2}-\frac{2}{21} \alpha_{H}^{3} \cos \left(3 \gamma_{H}\right) . ., B_{c}=1-\frac{1}{5} \alpha_{H}^{2}-\frac{1}{105} \alpha_{H}^{3} \cos \left(3 \gamma_{H}\right) . .
$$

This parameterization has been widely used to describe oblate nuclear ground states.

For the Bohr parameterization [17] the semiaxes are defined by

$$
\begin{aligned}
& a=R_{0}\left[1+\sqrt{5 / 4 \pi} \beta_{B} \cos \left(\gamma_{B}-\frac{2 \pi}{3}\right)\right], \\
& b=R_{0}\left[1+\sqrt{5 / 4 \pi} \beta_{B} \cos \left(\gamma_{B}+\frac{2 \pi}{3}\right)\right], \\
& c=R_{0}\left[1+\sqrt{5 / 4 \pi} \beta_{B} \cos \left(\gamma_{B}\right)\right] .
\end{aligned}
$$

so that in first order Bohr's parameters $\beta_{B}, \gamma_{B}$ are equal to Hill and Wheeler's parameters $\beta_{H}, \gamma_{H}$.

Recently, to describe precisely the nuclear ground-state shapes a "stretched" representation of the perturbed-spheroid parametrization has been introduced $[20,21]$. The stretched coordinates $\xi, \eta$ and $\zeta$ are defined by

$$
\begin{aligned}
\xi & =\sqrt{\frac{m \omega_{0}}{\hbar}\left[1-\frac{2}{3} \varepsilon_{2} \cos \left(\gamma+\frac{2}{3} \pi\right)\right]} x, \\
\eta & =\sqrt{\frac{m \omega_{0}}{\hbar}\left[1-\frac{2}{3} \varepsilon_{2} \cos \left(\gamma-\frac{2}{3} \pi\right)\right]} y, \\
\zeta & =\sqrt{\frac{m \omega_{0}}{\hbar}\left[1-\frac{2}{3} \varepsilon_{2} \cos (\gamma)\right]} z .
\end{aligned}
$$

and it follows the definitions of a stretched radius vector, a stretched polar angle and a stretched azimuthal angle. 


\subsection{Large deformations}

The Lawrence shapes defined, in cylindrical coordinates, by the quartic $\rho^{2}=$ $a z^{4}+b z^{2}+c$ has first been used to describe both the spherical, spheroidal constricted, scission and separated shapes $[1,22]$. These shapes have been generalized to asymmetric shapes [23] and given by

$$
\rho^{2}=R_{0}^{3} \lambda\left[z_{0}^{2}-\left(z+z_{s}\right)^{2}\right]\left[z_{2}\left|z_{2}\right|+\left(z+z_{s}-z_{1}\right)^{2}\right] .
$$

$z_{0}$ is the half-length, $z_{1}$ is the asymmetry parameter, $z_{1}=0$ for symmetric shapes. $z_{2}$ is the constriction or necking-in parameter and $z_{s}$ is introduced to fix the centre of mass at $z=0 . \lambda$ guarantees volume conservation. The particular cases: sphere, spheroids, scission and separated spheres occur respectively when $z_{0}=R_{0}$ and $z_{2} \rightarrow \infty, z_{0} \neq R_{0}$ and $z_{2} \rightarrow \infty, z_{2}=0, z_{2}<0$.

Using the dimensionless parameters $\zeta_{i}=z_{i} / R_{0}$ the geometric characteristics follow

$$
\begin{aligned}
& Q=\frac{8}{5} \pi \zeta_{0}^{5} \lambda\left[\frac{1}{7} \zeta_{0}^{2}+\frac{1}{3} \zeta_{2}^{2}-\frac{1}{3} \lambda\left(\frac{1}{21} \zeta_{0}^{4}+\frac{2}{7} \zeta_{0}^{2} \zeta_{2}^{2}+\zeta_{2}^{4}\right)\right], \\
& I_{\|}=\zeta_{0}^{5} \lambda\left(\frac{1}{21} \zeta_{0}^{4}+\frac{2}{7} \zeta_{0}^{2} \zeta_{2}^{2}+\zeta_{2}^{4}\right), \\
& I_{\perp}=\frac{1}{2}\left[I_{\|}+\zeta_{0}^{5} \lambda\left(\frac{3}{7} \zeta_{0}^{2}+\zeta_{2}^{2}\right)\right] .
\end{aligned}
$$

These shapes lead to rather elongated configurations with shallow necks and the scission configuration of two tangent spheres is not reproduced.

To describe fusion, scattering of two nuclei or compact fission, different matched surfaces of revolution have been proposed.

The two sphere parameterization for overlapping and separated fragments [24, 25, 26] is defined as

$$
\rho^{2}=r^{2}-(z-d)^{2} \text { for } z>0
$$

$\mathrm{d}$ is the distance from the centres of the separated or overlapping spheres to the origin. $\mathrm{r}$ is the maximal transverse distance. Assuming volume conservation the radius of the two spheres is $R=2^{-1 / 3} R_{0}$. The parameter $\varepsilon=1-d / r$ is used. $\varepsilon=1$ corresponds to a single sphere, $0<\varepsilon<1$ to two overlapping spheres, $\varepsilon=0$ to two tangent spheres and $\varepsilon<0$ to two separated spheres.

To study the exchange of nucleons between two fragments or nuclei the dumbbell parameterization has been defined in adding a cylindrical neck between two portions of a sphere $[26,27]$. This shape sequence describes the two touching sphere configuration but the derivatives of the curve are not continuous at the contact point of the cylinder and spheres.

The most flexible parameterization is the matched quadratic surfaces [1, 20, 21, 28, 29]. Three smoothly joined portions of quadratic surfaces of revolution are used : spheres, spheroids, hyperboloids, cylinders and cones. They are completely specified by

$$
\rho^{2}=a_{1}^{2}-\frac{a_{1}^{2}}{c_{1}^{2}}\left(z-l_{1}\right)^{2}, \quad l_{1}-c_{1} \leq z \leq z_{1},
$$




$$
\begin{aligned}
& \rho^{2}=a_{2}^{2}-\frac{a_{2}^{2}}{c_{2}^{2}}\left(z-l_{2}\right)^{2}, \quad z_{2} \leq z \leq l_{2}+c_{2}, \\
& \rho^{2}=a_{3}^{2}-\frac{a_{3}^{2}}{c_{3}^{2}}\left(z-l_{3}\right)^{2}, \quad z_{1} \leq z \leq z_{2} .
\end{aligned}
$$

The left-hand surface is denoted by 1 , the right-hand one by 2 and the middle one by 3 . Each surface is specified by the position of its center $l_{i}$, its transverse semiaxis $a_{i}$, and its semi-symmetry axis $c_{i}$. At the left and right intersections of the middle surface with the end surfaces the value of $\mathrm{z}$ is $\mathrm{z}_{1}$ and $\mathrm{z}_{2}$ respectively. After matching the surfaces and their derivatives it remains 6 parameters:

$$
\begin{aligned}
& \sigma_{1}=\left(l_{2}-l_{1}\right) / u, \quad \sigma_{2}=a_{3}^{2} / c_{3}^{2}, \quad \sigma_{3}=0.5\left[a_{1}^{2} / c_{1}^{2}+a_{2}^{2} / c_{2}^{2}\right], \\
& \alpha_{1}=0.5\left(l_{1}+l_{2}\right) / u, \quad \alpha_{2}=\left(a_{1}^{2}-a_{2}^{2}\right) / u^{2}, \quad \alpha_{3}=a_{1}^{2} / c_{1}^{2}-a_{2}^{2} / c_{2}^{2},
\end{aligned}
$$

where

$$
u=\sqrt{0.5\left(a_{1}^{2}+a_{2}^{2}\right)}
$$

A subset of the matched quadratic surfaces parameterization restricted to two spheres smoothly connected by a quadratic surface has also been employed [30].

A deformed two-centre shell model [31] has been developed mainly built from two ellipsoidally deformed Nilsson-type oscillators for axially symmetric shapes. The selected shape consists of two overlapping or not ellipsoids and a sphere rolling around the symmetry axis and being tangent to the two ellipsoids. Four geometric parameters define the shape: the two ratios of the ellipsoid semiaxes, the neck sphere radius and the distance between the fragment centres.

Recently [32] small and large deformations have been investigated within a Fourier expansion of deformed shapes

$$
\rho^{2}=R_{0}^{2} \sum_{n=1}^{\infty}\left[a_{2 n} \cos \left(\frac{(2 n-1) \pi}{2} \frac{z-z_{s h}}{z_{0}}\right)+a_{2 n+1} \sin \left(n \pi \frac{z-z_{s h}}{z_{0}}\right)\right] .
$$

The left and right ends of the shape are located at $z_{\min }=z_{s h}-z_{0}$ and $z_{\max }=z_{s h}+z_{0}$. The shift parameter $z_{s h}$ allows to replace the centre of the nuclear shape at the origin of the coordinate system. The parameters $a_{2}, a_{3}$ and $a_{4}$ describe the quadrupole, octupole and hexadecapole deformations or, equivalently, the elongation, reflection asymmetry and neck degree of freedom.

It is worthwhile to note that even though the approach within Hartree-FockBogoliubov theory and Gogny forces [33] is very different from the liquid drop model approach the idea is always to minimize the energy of the system via all the possible deformations and, mainly, the $Q_{2}, Q_{3}$ and $Q_{4}$ multipolar moments.

To describe ternary fission or quasi-molecular ternary excited states several configurations have been investigated. A restricted family of three colinearly aligned or triangle-like configurations has been defined but no simple formulas are available for the parameterization or for the liquid drop energies [34]. A shape consisting of three identical and aligned intersected spherical fragments or nuclei has also been used. The 
distance between the centers of the side fragments is the only degree of freedom, once the parent is fixed [35].

The ellipsoidal and two separated ellipsoid shapes, the elliptic lemniscatoids [36], the cassinian ovals [37, 38], the ternary shapes, the toroids [7, 39] and the bubbles $[8,39]$ that we have also used or defined will be more detailed in the following sections.

\section{Ellipsoidal shapes}

In polar coordinates the axially symmetric ellipsoids are defined by

$$
1 / R(\theta)^{2}=\sin ^{2} \theta / a^{2}+\cos ^{2} \theta / c^{2} .
$$

$a$ is the perpendicular semi-axis, $c$ being half the elongation on the revolution axis. The

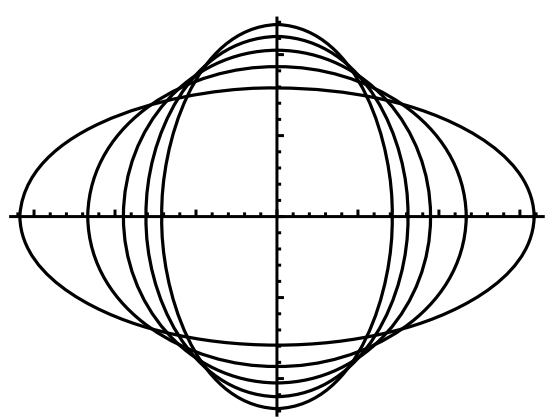

Figure 1. Ellipsoidal shapes versus the ratio $s=a / c$. The common revolution axis is the horizontal axis. $\mathrm{s}$ is respectively: $1 / 3,2 / 3,1,4 / 3$ and $5 / 3$. The ellipsoid is oblate when $s>1$ and prolate when $s<1$.

relation $a^{2} c=R_{0}^{3}$ ensures the volume conservation. $R_{0}$ is the radius of the sphere (see figure 1).

The related eccentricity is $e^{2}=1-s^{2}$ for the prolate shape while for the oblate case $e^{2}=1-s^{-2}[40,41,42]$.

For the prolate shape, the surface function is

$$
B_{s}=\frac{\left(1-e^{2}\right)^{1 / 3}}{2}\left[1+\frac{\sin ^{-1}(e)}{e\left(1-e^{2}\right)^{1 / 2}}\right]
$$

and for the oblate shape

$$
B_{s}=\frac{\left(1+\epsilon^{2}\right)^{1 / 3}}{2}\left[1+\frac{\ln \left(\epsilon+\left(1+\epsilon^{2}\right)^{1 / 2}\right)}{\epsilon\left(1+\epsilon^{2}\right)^{1 / 2}}\right] \quad \epsilon^{2}=s^{2}-1 .
$$

The relative Coulomb function reads in the prolate case

$$
B_{c}=\frac{\left(1-e^{2}\right)^{1 / 3}}{2 e} \ln \left(\frac{1+e}{1-e}\right)
$$

and for the oblate shape

$$
B_{c}=\frac{\left(1+\epsilon^{2}\right)^{1 / 3}}{\epsilon} \tan ^{-1} \epsilon
$$


For the prolate ellipsoidal shapes, $I$ and $Q$ are given by

$$
\begin{aligned}
& I_{\perp}=\frac{s^{-4 / 3}+s^{2 / 3}}{2}, \\
& I_{\|}=s^{2 / 3}, \\
& Q=\frac{8 \pi}{15}\left(s^{-4 / 3}-s^{2 / 3}\right) .
\end{aligned}
$$

\section{Two separated ellipsoid shapes}

For separated binary systems it may be necessary to introduce the ellipsoidal deformations (see figure 2) of the two objects (drops, nuclei, planets, galaxies,...).
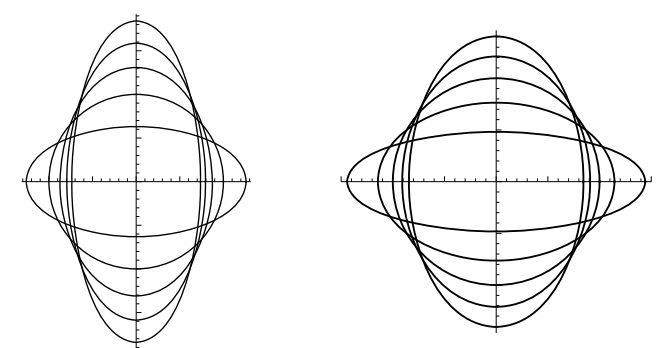

Figure 2. Two coaxial ellipsoid shapes.

The Coulomb (or gravitational) interaction energy between two coaxial ellipsoids is given by $[40,41,43,44,45]$

$$
E_{c, \text { int }}(r)=\frac{Q_{1} Q_{2}}{r}\left[s\left(\lambda_{1}\right)+s\left(\lambda_{2}\right)-1+S\left(\lambda_{1}, \lambda_{2}\right)\right] \quad \lambda_{i}^{2}=\frac{c_{i}^{2}-a_{i}^{2}}{r^{2}} .
$$

In the prolate case, $s\left(\lambda_{i}\right)$ is

$$
s\left(\lambda_{i}\right)=\frac{3}{4}\left(\frac{1}{\lambda_{i}}-\frac{1}{\lambda_{i}^{3}}\right) \ln \left(\frac{1+\lambda_{i}}{1-\lambda_{i}}\right)+\frac{3}{2 \lambda_{i}^{2}},
$$

and, for the oblate configurations,

$$
s\left(\lambda_{i}\right)=\frac{3}{2}\left(\frac{1}{\omega_{i}}+\frac{1}{\omega_{i}^{3}}\right) \tan ^{-1} \omega_{i}-\frac{3}{2 \omega_{i}^{2}} \quad \omega_{i}^{2}=-\lambda_{i}^{2} .
$$

$S\left(\lambda_{1}, \lambda_{2}\right)$ is expressed by a two-fold summation

$$
S\left(\lambda_{1}, \lambda_{2}\right)=\sum_{j=1}^{\infty} \sum_{k=1}^{\infty} \frac{3}{(2 j+1)(2 j+3)} \times \frac{3}{(2 k+1)(2 k+3)} \times \frac{(2 j+2 k) !}{(2 j) !(2 k) !} \lambda_{1}{ }^{2 j} \lambda_{2}{ }^{2 k} .
$$

\section{Elliptic lemniscatoids and asymmetric binary quasimolecules}

\subsection{Elliptic lemniscatoids}

The elliptic lemniscatoid shapes allows to describe continuously the transition from to two tangent equal spheres to one sphere or vice-versa via compact and creviced shapes 
with almost spherical ends, assuming volume conservation. In nuclear physics this shape sequence has been used to simulate, as a first approximation, the symmetric fusion and fission $[5,36]$.

Mathematically, the lemniscatoid is the first pedal surface of a prolate ellipsoid and the inverse of an oblate ellipsoid, the prolate ellipsoid being the reciprocal of the oblate ellipsoid (see figure 3).

The prolate ellipsoidal shape is expressed as

$$
x^{2} / a^{2}+y^{2} / a^{2}+z^{2} / c^{2}=1
$$

while its reciprocal oblate ellipsoid reads as

$$
a^{2} x^{2}+a^{2} y^{2}+c^{2} z^{2}=1 .
$$

The elliptic lemniscatoid is given by

$$
a^{2} x^{2}+a^{2} y^{2}+c^{2} z^{2}=\left(x^{2}+y^{2}+z^{2}\right)^{2} .
$$

The ratio $s=a / c$ of the neck radius to the half-elongation of the system defines

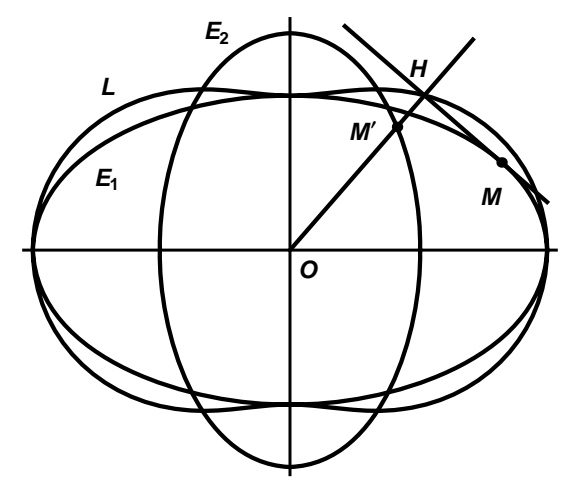

Figure 3. Relation between the elliptic lemniscatoid L and the ellipsoids $E_{1}$ and $E_{2}$. The point $\mathrm{H}$ of the lemniscatoid $\mathrm{L}$ is the projection of the point $\mathrm{O}$ onto the tangential plane to the prolate ellipsoid $E_{1}$ in M. The point M' of the oblate ellipsoid $E_{2}$ is the inverse of $\mathrm{H}$. The revolution axis is the horizontal axis.

completely the shape. When $\mathrm{s}$ increases from 0 to 1 the lemniscatoid varies continuously from two tangent spheres to a single one (see figure 4). When the perpendicular $\mathrm{x}$ axis is taken as axis of revolution the elliptic lemniscates generate pumpkin-like configurations (see section 9)).

In polar coordinates, the elliptic lemniscatoids are simply given by

$$
R(\theta)^{2}=a^{2} \sin ^{2} \theta+c^{2} \cos ^{2} \theta
$$

The volume and surface area follow

$$
V o l=\frac{4}{3} \pi R_{0}{ }^{3}=\frac{\pi}{12} c^{3}\left[4+6 s^{2}+\frac{3 s^{4}}{\sqrt{1-s^{2}}} \sinh ^{-1}\left(\frac{2}{s^{2}} \sqrt{1-s^{2}}\right)\right]
$$

and

$$
S=4 \pi R_{0}^{2} B_{s}=2 \pi c^{2}\left[1+\frac{s^{4}}{\sqrt{1-s^{4}}} \sinh ^{-1}\left(\frac{1}{s^{2}} \sqrt{1-s^{4}}\right)\right],
$$


$R_{0}$ being the radius of the spherical object.

$r$, the distance between the mass centres of the right and left parts of the object, is defined by

$$
r=2 \frac{\int_{0}^{c} z d^{3} r}{\int_{0}^{c} d^{3} r}
$$

For these elliptic lemniscatoids $r$ is simply given by

$$
r=\pi c^{4} \frac{1+s^{2}+s^{4}}{3 V}
$$

The perpendicular and parallel moments of inertia (relatively to the moment of inertia of the equivalent sphere $\frac{2}{5} m R_{0}^{2}$ ) are

$$
\begin{aligned}
& I_{\perp, r e l}=\frac{c^{5} s^{2}}{512\left(1-s^{2}\right) R_{0}^{5}}\left[\frac{112}{s^{2}}+8+30 s^{2}-135 s^{4}+\frac{120 s^{4}-135 s^{6}}{\sqrt{1-s^{2}}} \sinh ^{-1}\left(\sqrt{\frac{1-s^{2}}{s^{2}}}\right)\right], \\
& I_{\|, \text {rel }}=\frac{c^{5} s^{2}}{512\left(1-s^{2}\right) R_{0}^{5}}\left[\frac{32}{s^{2}}+48+100 s^{2}-210 s^{4}+\frac{240 s^{4}-210 s^{6}}{\sqrt{1-s^{2}}} \sinh ^{-1}\left(\sqrt{\frac{1-s^{2}}{s^{2}}}\right)\right] .
\end{aligned}
$$

The dimensionless quadrupole moment $Q$ is

$$
Q=\frac{\pi c^{5} s^{2}}{96\left(1-s^{2}\right) R_{0}^{5}}\left[\frac{16}{s^{2}}-8-14 s^{2}+15 s^{4}-\frac{24 s^{4}-15 s^{6}}{\sqrt{1-s^{2}}} \sinh ^{-1}\left(\sqrt{\frac{1-s^{2}}{s^{2}}}\right)\right] .
$$

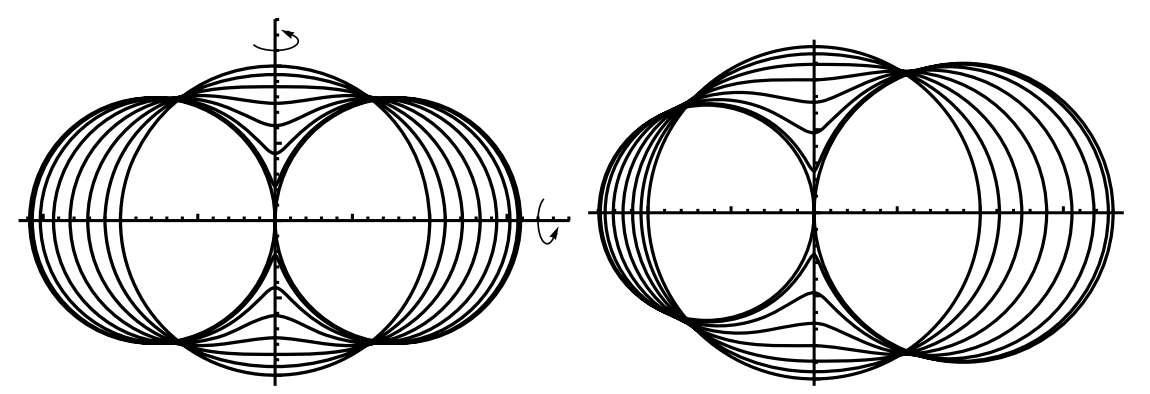

Figure 4. On the left, evolution of the elliptic lemniscatoids versus the ratio $s=a / c$. The shape varies continuously from two spheres $(s=0)$ to one sphere $(s=1)$. The neck exists for $s<0.5 \sqrt{2}$. On the right, two halves of different lemniscatoids of same perpendicular distance a are linked in the transverse plane.

\subsection{Asymmetric binary quasimolecules}

To describe the path from one sphere to two different spheres in contact or vice versa (see figure 4) two halves of different elliptic lemniscatoids may be connected in the transverse plane assuming the same transversal radius. These shape sequence allows the study of the alpha emission or capture and cluster radioactivity $[9,10,11,46]$, the asymmetric fusion $[3,4]$ and the asymmetric fission through compact and creviced shapes [6]. 
Mathematically the shape is defined by

$$
R(\theta)^{2}= \begin{cases}a^{2} \sin ^{2} \theta+c_{1}{ }^{2} \cos ^{2} \theta & 0 \leq \theta \leq \pi / 2 \\ a^{2} \sin ^{2} \theta+c_{2}{ }^{2} \cos ^{2} \theta & \pi / 2 \leq \theta \leq \pi .\end{cases}
$$

$c_{1}$ and $c_{2}$ are the two half-elongations on the $\mathrm{z}$ axis.

The relation $R_{0}{ }^{3}=R_{1}{ }^{3}+R_{2}{ }^{3}$ ensured the volume conservation, $R_{0}, R_{1}$ and $R_{2}$ being the radii of the total and different spheres.

The two parameters $s_{1}=a / c_{1}$ and $s_{2}=a / c_{2}$ define completely the quasimolecular shape. The two radii $R_{1}$ and $R_{2}$ allows to link $s_{1}$ and $s_{2}$ :

$$
s_{2}^{2}=\frac{s_{1}^{2}}{s_{1}^{2}+\left(1-s_{1}^{2}\right)\left(R_{2} / R_{1}\right)^{2}} .
$$

When $s_{1}$ increases from 0 to 1 the shape varies from to two touching unequal spheres to one sphere. The volume conservation of the two parts is assumed. The separation plane is at a distance $z_{v}$ from the origin. $r_{1}$ and $r_{2}$ are calculated from the quantity $z_{v}$, solution of the equation

$$
\begin{aligned}
& \frac{1}{3} z^{3}-\frac{1}{2} a^{2} z+\frac{1}{12}\left(2 c_{2}{ }^{3}+3 a^{2} c_{2}\right)+ \\
& \frac{1}{2} \sqrt{c_{2}{ }^{2}-a^{2}}\left[D^{2} \sinh ^{-1}\left(\frac{c_{2}}{D}\right)-D^{2} \sinh ^{-1}\left(\frac{z}{D}\right)-z \sqrt{z^{2}+D^{2}}\right]=\frac{4}{3} R_{2}{ }^{3}
\end{aligned}
$$

with $D^{2}=a^{4} / 4\left(c_{2}^{2}-a^{2}\right)$.

The positions $r_{1}$ and $r_{2}$ are then obtained by

$$
\begin{aligned}
r_{1}=\frac{1}{\frac{4}{3} R_{1}^{3}}\left\{\frac{z_{v}{ }^{4}-a^{2} z_{v}{ }^{2}}{4}+\frac{c_{1}^{4}+a^{2} c_{1}{ }^{2}+a^{4}}{12}-\right. & \\
& \left.\frac{a s_{2}{ }^{2}}{3\left(1-s_{2}{ }^{2}\right)}\left[\left(\frac{z_{v}{ }^{2}\left(1-s_{2}{ }^{2}\right)}{s_{2}{ }^{2}}+\frac{a^{2}}{4}\right)^{3 / 2}-\frac{a^{3}}{8}\right]\right\}
\end{aligned}
$$

and

$$
\begin{aligned}
r_{2}= & \frac{1}{\frac{4}{3} R_{2}{ }^{3}}\left\{\frac{z_{v}{ }^{4}-a^{2} z_{v}{ }^{2}}{4}-\frac{a^{4}}{4}\left(\frac{1-s_{2}{ }^{2}}{s_{2}{ }^{4}}\right)+\right. \\
& \left.\frac{a s_{2}{ }^{2}}{3\left(1-s_{2}{ }^{2}\right)}\left[a^{3}\left(\frac{1}{s_{2}{ }^{2}}-\frac{1}{2}\right)^{3}-\left(\frac{z_{v}{ }^{2}\left(1-s_{2}{ }^{2}\right)}{s_{2}{ }^{2}}+\frac{a^{2}}{4}\right)^{3 / 2}\right]\right\} .
\end{aligned}
$$

The distance $r$ between the mass centres of the right and left parts of the physical object is $r=r_{1}+r_{2}$.

In nuclear physics the search for superheavy nuclei and new very asymmetric decay has renewed interest in the understanding and description of the alpha decay and cluster radioactivity. Since the pioneering work of Viola and Seaborg [47] several models have been developed and regularly improved $[48,49,50,51,52,53,54,55,56$, $57,58,59,60,61,62,63,64,65,66,67,68,69,70,71,72,73,74,75]$ and analytic expressions have been proposed to calculate rapidly the alpha and cluster decay halflives. Comparisons between these models and their predictive capacities may be found in Ref. [68, 69, 73, 74]. 


\section{Hyperbolic lemniscatoids}

The shape of a viscous object at the scission point depends strongly on the mean free path of its constituants and its viscosity. The scission may occur through compact and creviced shapes or through shallow and elongated shapes. This last deformation valley can be simulated within hyperbolic lemniscatoids (Cassinian ovaloids) [37, 38].

For the one-body configurations the hyperbolic lemniscatoids are expressed as

$$
x^{2}=-z^{2}+0.5 c^{2}\left(s^{2}-1\right)+0.5 c \sqrt{8\left(1-s^{2}\right) z^{2}+c^{2}\left(1+s^{2}\right)^{2}} .
$$

For the two-body shapes the ovals are defined by

$$
x^{2}=-z^{2}-0.5 c^{2}\left(s^{2}+1\right)+0.5 c \sqrt{8\left(1+s^{2}\right) z^{2}+c^{2}\left(1-s^{2}\right)^{2}} .
$$

These shapes are one-parameter dependent when the volume conservation is assumed. For the one-body shapes the ratio $s=a / c$ of the minor and major axes can still be used. For the two-body configurations, the opposite s of the ratio of the distance between the tips of the fragments and the system elongation has been selected (see figure 5 and [38]). When s evolves from -1 to 1 the shapes varies from two infinitely separated and identical spherical objects to one sphere.

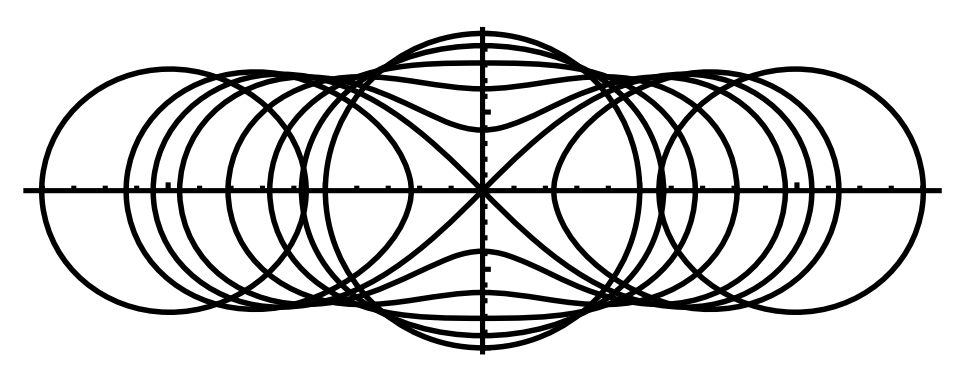

Figure 5. Cassinian ovals versus the parameter s. At the scission point, the configuration is the Bernoulli lemniscate. The development of a neck begins when $s<\sqrt{3} / 3$.

For the one-body shape the volume of the system is

$$
V o l=\frac{\pi c^{3}}{12}\left[-2+6 s^{2}+3 \frac{\left(1+s^{2}\right)^{2}}{\sqrt{2\left(1-s^{2}\right)}} \sinh ^{-1}\left(\frac{2 \sqrt{2\left(1-s^{2}\right)}}{1+s^{2}}\right)\right]
$$

and for the two-body shapes

$$
V o l=\frac{\pi c^{3}}{12}\left[-2(1+s)^{3}+3 \frac{\left(1-s^{2}\right)^{2}}{\sqrt{2\left(1+s^{2}\right)}} \sinh ^{-1}\left(\frac{2(1+s) \sqrt{2\left(1+s^{2}\right)}}{(1-s)^{2}}\right)\right]
$$

For the one-body shapes, the surface function reads

$$
\begin{array}{r}
B_{s}=\frac{c^{2}}{4 R_{0}^{2}} \times\left[4\left(1+s^{2}\right)+2 \sqrt{\frac{2\left(1+s^{2}\right)}{1-s^{2}}} s^{2} F\left(\sin ^{-1} \sqrt{1-s^{2}}, \frac{1}{\sqrt{1+s^{2}}}\right)\right. \\
\left.-2\left(1+s^{2}\right) \sqrt{\frac{2\left(1+s^{2}\right)}{1-s^{2}}} E\left(\sin ^{-1} \sqrt{1-s^{2}}, \frac{1}{\sqrt{1+s^{2}}}\right)\right],
\end{array}
$$


$\mathrm{E}$ and $\mathrm{F}$ being incomplete elliptic integrals. $B_{s}$ is calculated numerically for the twobody configuration.

The distance $r$ between the centres of the right and left parts is for the one and two-body shapes

$$
\begin{aligned}
& r=\frac{c^{4}}{8 R_{0}^{3}} \times\left(1+4 s^{2}+s^{4}\right), \\
& r=\frac{c^{4}}{8 R_{0}^{3}} \times \frac{\left(1-s^{2}\right)^{3}}{1+s^{2}} .
\end{aligned}
$$

For the one-body shapes the parallel and perpendicular moments of inertia are given by

$$
\begin{aligned}
& I_{\|}=\frac{c^{5}}{512\left(1-s^{2}\right) R_{0}{ }^{5}} \times \\
& {\left[147-27 s^{2}-15 s^{4}-225 s^{6}-\frac{15\left(1+s^{2}\right)^{2}\left(15-34 s^{2}+15 s^{4}\right)}{2 \sqrt{2\left(1-s^{2}\right)}} \sinh ^{-1}\left(\frac{2 \sqrt{2\left(1-s^{2}\right)}}{1+s^{2}}\right)\right],} \\
& I_{\perp}=\frac{c^{5}}{1024\left(1-s^{2}\right) R_{0}{ }^{5}} \times \\
& {\left[269+251 s^{2}-145 s^{4}-255 s^{6}-\frac{15\left(1+s^{2}\right)^{2}\left(17-30 s^{2}+17 s^{4}\right)}{2 \sqrt{2\left(1-s^{2}\right)}} \sinh ^{-1}\left(\frac{2 \sqrt{2\left(1-s^{2}\right)}}{1+s^{2}}\right)\right] .}
\end{aligned}
$$

The quadrupole moment is defined by

$$
\begin{aligned}
& Q=\frac{\pi c^{5}}{192\left(1-s^{2}\right) R_{0}{ }^{5}} \times \\
& {\left[-5+61 s^{2}-23 s^{4}+39 s^{6}+\frac{3\left(1+s^{2}\right)^{2}\left(13-38 s^{2}+13 s^{4}\right)}{2 \sqrt{2\left(1-s^{2}\right)}} \sinh ^{-1}\left(\frac{2 \sqrt{2\left(1-s^{2}\right)}}{1+s^{2}}\right)\right] .}
\end{aligned}
$$

For the two-body shapes these quantities are expressed as

$$
\begin{aligned}
I_{\|}= & \frac{c^{5}}{512\left(1+s^{2}\right) R_{0}{ }^{5}} \times\left[147+225 s+27 s^{2}-15 s^{3}-15 s^{4}+27 s^{5}+225 s^{6}+147 s^{7}\right. \\
& \left.-\frac{15\left(1-s^{2}\right)^{2}\left(15+34 s^{2}+15 s^{4}\right)}{2 \sqrt{2\left(1+s^{2}\right)}} \sinh ^{-1}\left(\frac{2(1+s) \sqrt{2\left(1+s^{2}\right)}}{(1-s)^{2}}\right)\right] \\
I_{\perp}= & \frac{c^{5}}{1024\left(1+s^{2}\right) R_{0}{ }^{5}} \times\left[269+255 s-251 s^{2}-145 s^{3}-145 s^{4}-251 s^{5}+255 s^{6}\right. \\
& \left.+269 s^{7}-\frac{15\left(1-s^{2}\right)^{2}\left(17+30 s^{2}+17 s^{4}\right)}{2 \sqrt{2\left(1+s^{2}\right)}} \sinh ^{-1}\left(\frac{2(1+s) \sqrt{2\left(1+s^{2}\right)}}{(1-s)^{2}}\right)\right] \\
Q= & \frac{\pi c^{5}}{192\left(1-s^{2}\right) R_{0}{ }^{5}} \times\left[-5-39 s-61 s^{2}-23 s^{3}-23 s^{4}-61 s^{5}-39 s^{6}-5 s^{7}\right. \\
& \left.+\frac{3\left(1-s^{2}\right)^{2}\left(13+38 s^{2}+13 s^{4}\right)}{2 \sqrt{2\left(1+s^{2}\right)}} \sinh ^{-1}\left(\frac{2(1+s) \sqrt{2\left(1+s^{2}\right)}}{(1-s)^{2}}\right)\right]
\end{aligned}
$$




\section{Comparison between the elliptic lemniscatoid and hyperbolic lemniscatoid shape valleys}

The elliptic lemniscatoid shape sequence describes the rapid transition from one sphere to two tangent spheres or vice versa and to the rapid formation and rupture of a neck in one-body systems while keeping almost spherical ends or the rapid coalescence of almost spherical separated systems. The hyperbolic lemniscatoid shape sequence describes a deformation where the elongation occurs before the constriction.

The quadrupole moment and the perpendicular moment of inertia which are the main available geometrical experimental data are similar in the two paths. In contrast the parallel and effective moment of inertia are quite different in the two valleys (see figure 6 and [38]) but their values are difficult to obtain experimentally (via the angular distribution of the fragments). So it is not easy from the experimental data to know the real path investigated in nuclear reactions at low energies.
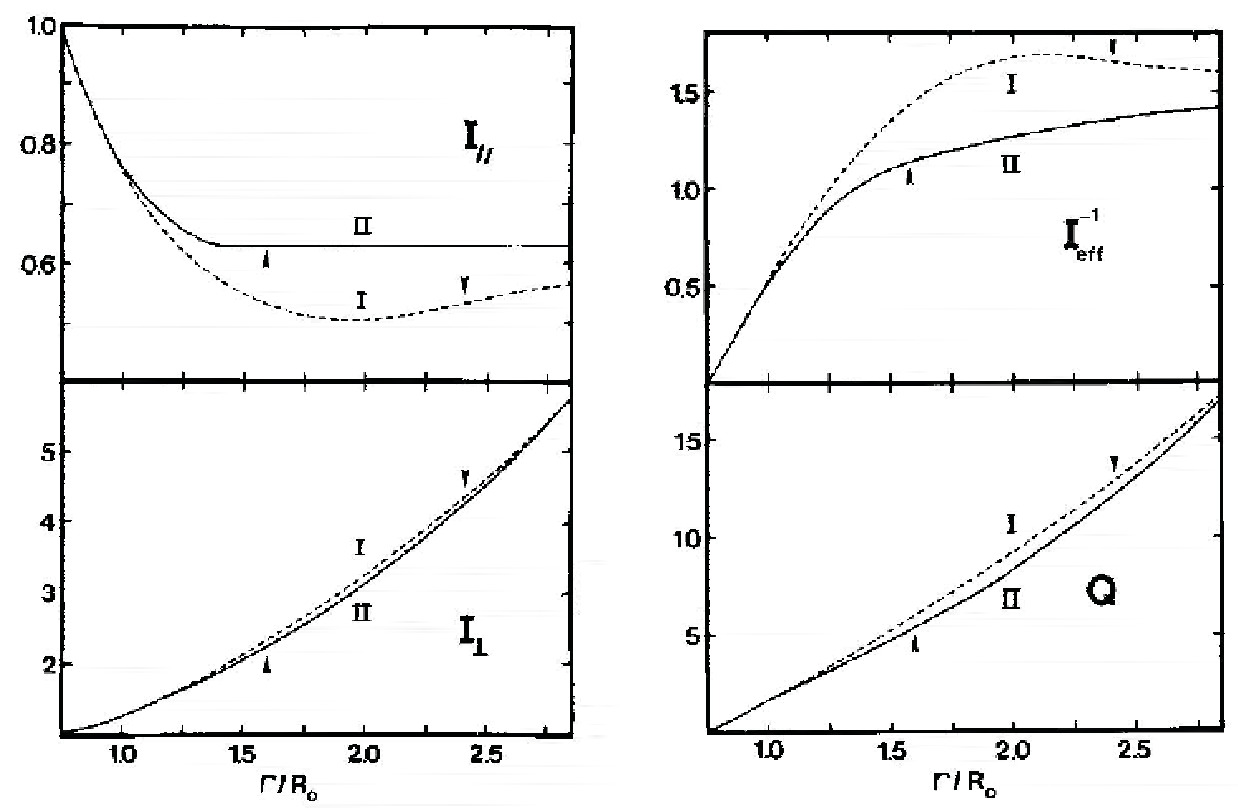

Figure 6. Relative parallel, perpendicular and inverse effective moments of inertia and quadrupole moment for the hyperbolic (path I) and elliptic (path II) lemniscatoid shape paths.

The different possible shapes taken by a fissioning nucleus were firstly explore using a development of the radius in terms of Legendre polynomials, thinking that the fission process is only governed by the balance between the repulsive Coulomb forces and the attractive surface tension forces. This method leads naturally to smooth elongated one-body configurations resembling to hyperbolic lemniscatoids. Later on, it appears that, within liquid drop models, to reproduce the fusion data and the alpha and cluster radioactivities one must introduce an additional term, the so-called proximity energy, to take into account the strong nuclear interaction between nucleons of the surfaces in 
regard between almost spherical nuclei or in a deep crevice in one-body shapes. This proximity energy is evidently very small for elongated shapes with shallow necks and, then, it is often neglected. In contrast this term is essential to describe the fusion or fission through compact and creviced shapes. It has been shown $[5,38]$ that it exists a degeneracy in energy between these two deformation valleys. These two paths implicitly seem to correspond to different values of the nuclear viscosity (diabaticity or adiabaticity ?) and then different dynamics and different degrees of thermalization. The dependence of the nuclear viscosity on the temperature, angular momentum, isospin,... obtained from fission experiments may be found in [76].

\section{Prolate symmetric and asymmetric ternary shapes}

\subsection{Prolate symmetric ternary shapes}

Assuming volume conservation, prolate symmetric ternary shapes varying from three aligned tangent identical spheres to one sphere or vice versa can be described from elliptic lemniscatoids. Such configurations may simulate several excited molecular states of ${ }^{12} C$ formed from three $\alpha$ particles during the stellar nucleosynthesis. To define these ternary quasimolecular shapes, the cartesian coordinates are preferable to the polar coordinates since the symmetry plane does not correspond to the neck planes.

In the first quadrant the equation defining these shapes is (see figure 7 and [77])

$$
x^{2}=0.5\left[a^{2}-2(z-d)^{2}+\sqrt{a^{4}+4(z-d)^{2}\left(c^{2}-a^{2}\right)}\right] .
$$

a and $\mathrm{c}$ are respectively the neck radius and half the elongation of the corresponding binary shape. The parameter $s=a / c$ is always sufficient to define the shape. $\mathrm{d}$ is the distance between the transverse $\mathrm{x}$ axis and the position of the crevice, namely

$$
d= \begin{cases}0.5 c \sqrt{\frac{1-2 s^{2}}{1-s^{2}}} & \text { for } 0 \leq s<0.5 \sqrt{2} \\ 0 & \text { for } 0.5 \sqrt{2} \leq s \leq 1\end{cases}
$$

The maximal perpendicular distance is given by

$$
h_{\text {max }}= \begin{cases}0.5 c /\left(1-s^{2}\right)^{1 / 2} & \text { when } 0 \leq s<0.5 \sqrt{2} \\ a & \text { when } 0.5 \sqrt{2} \leq s \leq 1\end{cases}
$$

The volume is given by

$$
V o l=\frac{4}{3} \pi R_{0}^{3}=\frac{\pi c^{3}}{12}\left[4+6 s^{2}+g(\alpha)+\frac{3 s^{4}}{\sqrt{1-s^{2}}} \ln \left(\frac{2-s^{2}+2 \sqrt{1-s^{2}}}{h(\alpha)}\right)\right],
$$

$R_{0}$ being the radius of the total sphere, $\alpha=d / c$ and

$$
g(\alpha)= \begin{cases}6 \alpha+6 \alpha s^{2}-8 \alpha^{3} & \text { for } 0 \leq s<0.5 \sqrt{2} \\ 0 & \text { for } 0.5 \sqrt{2} \leq s \leq 1\end{cases}
$$

and

$$
h(\alpha)= \begin{cases}-2 \alpha \sqrt{1-s^{2}}+1-s^{2} & \text { when } 0 \leq s \leq 0.5 \sqrt{2} \\ s^{2} & \text { when } 0.5 \sqrt{2} \leq s \leq 1\end{cases}
$$


The dimensionless surface function is

$$
B_{s}= \begin{cases}\frac{c^{2}}{2 R_{0}^{2}}\left[1+\frac{\sqrt{1-2 s^{2}}}{2}+\frac{s^{4}}{\sqrt{1-s^{4}}} \ln \left(\frac{\sqrt{2}\left(1+\sqrt{1-s^{4}}\right)}{\sqrt{1-s^{2}}-\sqrt{\left(1+s^{2}\right)\left(1-2 s^{2}\right)}}\right)\right] & \text { for } 0 \leq s \leq 0.5 \sqrt{2} \\ \frac{c^{2}}{2 R_{0}^{2}}\left[1+\frac{s^{4}}{\sqrt{1-s^{4}}} \ln \left(\frac{1+\sqrt{1-s^{4}}}{s^{2}}\right)\right] & \text { for } 0.5 \sqrt{2} \leq s<1 .\end{cases}
$$

The distance between the right and left parts of the system is expressed as

$$
r= \begin{cases}c\left(2 \alpha+\frac{\pi c^{3}\left(11-8 s^{2}\right)}{48 V\left(1-s^{2}\right)^{2}}\right) & \text { when } 0 \leq s<0.5 \sqrt{2} \\ \frac{\pi c^{4}}{3 V}\left(1+s^{2}+s^{4}\right) & \text { when } 0.5 \sqrt{2} \leq s \leq 1\end{cases}
$$

When the three spheres are separated, the total length of the shape is

$$
l=1.5 r+1.625 R_{1},
$$

the radius of a small sphere being $R_{1}=3^{-1 / 3} R_{0}$.
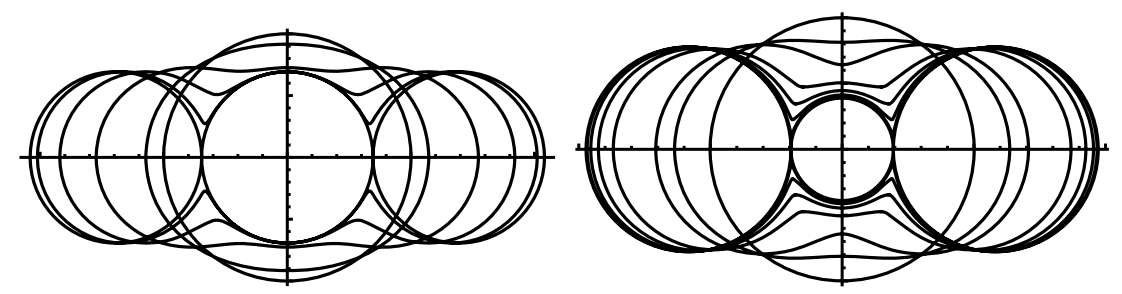

Figure 7. On the left, prolate and symmetric ternary quasimolecular shapes. The shape varies from three touching equal and aligned spheres $(s=0)$ to one sphere $(s=1)$. The neck disappears when $s>\sqrt{2} / 2$. On the right, quasimolecular shapes varying from one sphere to two equal spheres in contact with a central smaller sphere. The ratio between the radii is 0.5 .

\subsection{Prolate asymmetric ternary shapes}

To describe more asymmetric ternary configurations (see figure 7) and [78, 79]) from the asymmetric binary quasimolecular shapes (see section 5) it is sufficient to cut the smallest fragment at its maximal perpendicular distance by a plane perpendicular to the axis of revolution and to introduce a mirror symmetry. Then, the two parameters $s_{1}=a / c_{1}$ and $s_{2}=a / c_{2}$ define completely the shape mathematically. $a$ is the neck radius. $c_{1}$ and $c_{2}$ are respectively the elongations of the external and central fragments. The one sphere configuration correspond to $s_{1}=s_{2}=1$. For $s_{1}=s_{2}=0$ two equal spheres of radius $R_{1}$ touch a central smaller sphere of radius $R_{2} . s_{1}$ and $s_{2}$ may be linked by the expression

$$
s_{2}^{2}=\frac{s_{1}^{2}}{s_{1}^{2}+\left(1-s_{1}^{2}\right)\left(R_{2} / R_{1}\right)^{2}}
$$

and consequently

$$
c_{2}^{2}=c_{1}^{2}\left[s_{1}^{2}+\left(1-s_{1}^{2}\right)\left(R_{2} / R_{1}\right)^{2}\right]
$$

In the first quadrant the shape is defined by

$$
x^{2}=-(z-d)^{2}+0.5 s_{i}{ }^{2} c_{i}^{2}+0.5 c_{i} \sqrt{4\left(1-s_{i}{ }^{2}\right)(z-d)^{2}+s_{i}{ }^{4} c_{i}^{2}}
$$


$(i=1$ for $z>d$ and $i=2$ for $z<d)$.

The volume is given by

$$
\begin{aligned}
V o l= & \frac{\pi c_{1}^{3}}{12}\left[4+6 s_{1}{ }^{2}+\frac{3 s_{1}{ }^{4}}{\sqrt{1-s_{1}^{2}}} \sinh ^{-1}\left(\frac{2 \sqrt{1-s_{1}{ }^{2}}}{s_{1}{ }^{2}}\right)\right] \\
& +\frac{\pi c_{2}^{3}}{12}\left[6 \alpha+6 \alpha s_{2}{ }^{2}-8 \alpha^{3}+\frac{3 s_{2}{ }^{4}}{\sqrt{1-s_{2}^{2}}} \sinh ^{-1}\left(\frac{2 \alpha \sqrt{1-s_{2}{ }^{2}}}{s_{2}{ }^{2}}\right)\right]
\end{aligned}
$$

where $\alpha=d / c_{2}$.

The surface $S$ reads

$$
\begin{aligned}
S=2 & \pi c_{1}{ }^{2}\left[1+\frac{s_{1}{ }^{4}}{\sqrt{1-s_{1}^{4}}} \sinh ^{-1}\left(\frac{\sqrt{1-s_{1}^{4}}}{s_{1}^{2}}\right)\right] \\
& +2 \pi c_{2}{ }^{2}\left[\alpha \sqrt{1-s_{2}^{2}}+\frac{s_{2}{ }^{4}}{\sqrt{1-s_{2}^{4}}} \sinh ^{-1}\left(\frac{\alpha \sqrt{2\left(1-s_{2}^{4}\right)}}{s_{2}^{2}}\right)\right] .
\end{aligned}
$$

The distance between the mass centres of the right and left parts is

$$
r=\frac{\pi c_{1}^{4}}{3 V}\left(1+s_{1}{ }^{2}+s_{1}^{4}\right)+c_{2}\left[2 \alpha+\frac{\pi c_{2}^{3} \alpha^{4}}{3 V} \cdot \frac{-5+8 s_{2}{ }^{2}+16 s_{2}{ }^{6}-16 s_{2}{ }^{8}}{\left(1-2 s_{2}^{2}\right)^{2}}\right] .
$$

When the three spheres are separated :

$$
\begin{aligned}
& B_{s}=\frac{2+\left(R_{2} / R_{1}\right)^{2}}{\left(2+\left(R_{2} / R_{1}\right)^{3}\right)^{2 / 3}} \\
& r=\frac{3}{2+\left(R_{2} / R_{1}\right)^{3}}\left(\frac{\left(R_{2} / R_{1}\right)^{4} R_{1}}{4}+\frac{4}{3} D\right) .
\end{aligned}
$$

$2 D$ is the distance between the centres of the two equal external spheres.

\section{More exotic shapes}

Experimentally, very distorted configurations have been observed in nuclear physics, astrophysics... and some of them can be roughly approximated by toroidal shapes and bubbles. For example, in heavy-ion reactions at Fermi energies, complicated shapes intermediate between toroids and bubbles have been indirectly detected [80, 81]. These systems are often transient states formed after a violent collision and evolving towards pumpkin-like shapes and, later on, tori and n-spheres emitted in the same plane or evolving to bubbles splitting in the whole space.

A pumpkin-like shape may be generated by elliptic lemniscates (see figure 4) in taking the vertical axis as axis of revolution (see figure 8). The dimensionless parameter $s=a / c$ is always sufficient to characterize the shape. When $s$ diminishes from 1 to 0 , an hollow progressively appears. The shape varies from a sphere to a ring torus for which the upper and lower hollows are connected together. Later on, this initial configuration evolves to a torus with large radius $r_{t}$. The dimensionless parameter $s_{t}$ allows to follow this evolution $[7,8]$

$$
s_{t}=\left(r_{t}-r_{s}\right) / 2 r_{s},
$$

$r_{s}$ and $r_{t}$ being the sausage and torus radii. 

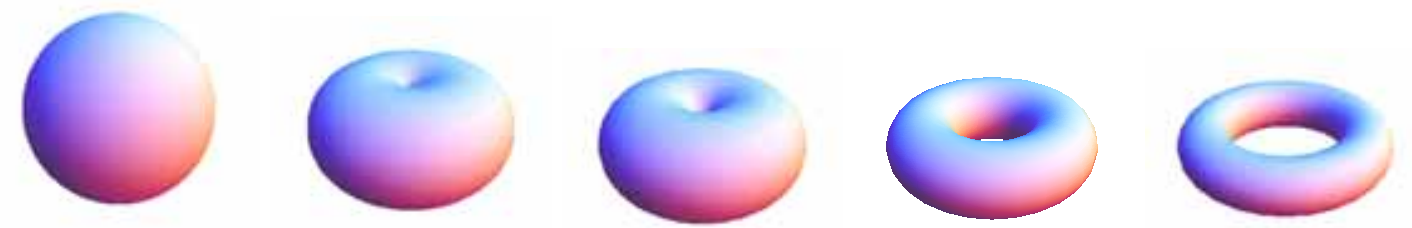

Figure 8. Pumpkin-like configuration versus the ratio s of the semi-axes and torus defined by the sausage $r_{s}$ and torus $r_{t}$ radii. The axis of revolution is the vertical axis.

\subsection{Pumpkin-like shapes}

The volume, moment of inertia, mean square radius and surface function are given by

$$
\begin{gathered}
V o l=\frac{4 \pi R_{0}^{3}}{3}=\frac{4 \pi c^{3}}{3}\left[\frac{s^{3}}{4}+\frac{3}{8}\left(s+\frac{\sin ^{-1}\left(\sqrt{1-s^{2}}\right)}{\sqrt{1-s^{2}}}\right)\right] \\
I_{\perp, r e l}=\frac{3 c^{5}}{2 R_{0}^{5}\left(1-s^{2}\right)}\left(-\frac{s^{7}}{24}-\frac{s^{5}}{16}-\frac{25 s^{3}}{192}+\frac{35 s}{128}-\frac{5}{16}\left(s^{2}-\frac{7}{8}\right) \frac{\sin ^{-1}\left(\sqrt{1-s^{2}}\right)}{\sqrt{1-s^{2}}}\right) \\
\left\langle r^{2}\right\rangle_{r e l}=\frac{\left\langle r^{2}\right\rangle}{\frac{3}{5} R_{0}^{2}}=\frac{5 c^{5}}{4 R_{0}^{5}}\left[\frac{2 s^{5}}{15}+\frac{s^{3}}{6}+\frac{1}{4}\left(s+\frac{\sin ^{-1}\left(\sqrt{1-s^{2}}\right)}{\sqrt{1-s^{2}}}\right)\right] \\
B_{s}=\frac{s}{4 \pi R_{0}^{2}}=\frac{c^{2}}{2 R_{0}^{2}}\left(s^{2}+\frac{\sin ^{-1}\left(\sqrt{1-s^{4}}\right)}{\sqrt{1-s^{4}}}\right) .
\end{gathered}
$$

\subsection{Ring torus}

For a formed torus these quantities are given by

$$
\begin{aligned}
& V o l=\frac{4 \pi R_{0}^{3}}{3}=2 \pi^{2} r_{t} r_{s}^{2}=\frac{\pi^{2} c_{t}^{3}}{4}\left(1+2 s_{t}\right), \\
& I_{\perp, r e l}=\frac{35}{32}\left(1+3 s_{t}+3 s_{t}^{2}\right)\left(\frac{16}{3 \pi\left(1+2 s_{t}\right)}\right)^{2 / 3}, \\
& \left\langle r^{2}\right\rangle_{r e l}=\frac{5}{6}\left(1+2 s_{t}+2 s_{t}^{2}\right)\left(\frac{16}{3 \pi\left(1+2 s_{t}\right)}\right)^{2 / 3}, \\
& B_{s}=\frac{4 \pi^{2} r_{s} r_{t}}{4 \pi R_{0}^{2}}=\frac{\pi c_{t}^{2}}{4 R_{0}^{2}}\left(1+2 s_{t}\right),
\end{aligned}
$$

and finally

$$
r_{s}=R_{0}\left(\frac{2}{3 \pi(1+2 s)}\right)^{1 / 3} .
$$




\subsection{Bubbles}

Bubbles of thick skin are often formed within violent reactions where the out of equilibrium effects play an essential role. Calculations within bubbles of constant density can give a first rough approach of the more complex reality [8]. Assuming volume conservation, the bubble characteristics can be calculated from the ratio $p=r_{1} / r_{2}$ of the inner and outer radii $r_{1}$ and $r_{2}$.

$$
\begin{aligned}
& V o l=\frac{4 \pi R_{0}^{3}}{3}=\frac{4 \pi}{3}\left(r_{2}^{3}-r_{1}^{3}\right), \\
& r_{1}=R_{0} p\left(1-p^{3}\right)^{-1 / 3} \\
& r_{2}=R_{0}\left(1-p^{3}\right)^{-1 / 3} .
\end{aligned}
$$

The relative root mean square radius reads

$$
\left\langle r^{2}\right\rangle_{\text {rel }}{ }^{1 / 2}=\frac{\left\langle r^{2}\right\rangle^{1 / 2}}{\sqrt{3 / 5} R_{0}}=\left(1-p^{5}\right)^{1 / 2}\left(1-p^{3}\right)^{-5 / 6}
$$

The surface, Coulomb and moment of inertia functions are

$$
\begin{aligned}
& B_{s}=\frac{1+p^{2}}{\left(1-p^{3}\right)^{2 / 3}}, \\
& B_{c}=\frac{1-2.5 p^{3}+1.5 p^{5}}{\left(1-p^{3}\right)^{5 / 3}}, \\
& I_{\perp, \text { rel }}=\left(1-p^{5}\right)\left(1-p^{3}\right)^{-5 / 3} .
\end{aligned}
$$

\section{Summary}

In a first part, most of the geometrical shapes defined to model the nuclear states and reactions (alpha decay, cluster radioactivity, fusion, fission and fragmentation) are briefly reviewed. Later on, the shape sequences that we have used previously, mainly formed from generalized lemniscatoids, are more extensively detailed. Their geometric characteristics are given: root mean square radius, volume, surface and Coulomb energies as well as their moments of inertia and quadrupole moments. These shapes allow to describe the transitions from one compact configuration to several ones or vice versa and may be perhaps useful also to describe physical objects such as drops, droplets and fluids in chemistry, mechanics, astrophysics, hydrodynamics,...

\section{References}

[1] Hasse R W and Myers W D 1988 Geometrical Relationships of Macroscopic Nuclear Physics (Berlin: Springer)

[2] Royer G, Mokus N and Jahan J 2017 Phys. Rev. C 95054610

[3] Royer G and Remaud B 1985 Nucl. Phys. A 444477

[4] Royer G 1986 J. Phys. G: Nucl. Part. Phys. 12623 
[5] Royer G and Remaud B 1984 J. Phys. G: Nucl. Part. Phys. 101057

[6] Royer G, Jaffré M and Moreau D 2012 Phys. Rev. C 86044326

[7] Fauchard C and Royer G 1996 Nucl. Phys. A 598125

[8] Royer G, Haddad F and Jouault B 1996 Nucl. Phys. A 605403

[9] Royer G 2000 J. Phys. G: Nucl. Part. Phys. 261149

[10] Royer G 2010 Nucl. Phys. A 848279

[11] Royer G and Moustabchir R 2001 Nucl. Phys. A 683182

[12] Royer G, Ramasamy G and Eudes P 2015 Phys. Rev. C 92054308

[13] Beringer R and Knox W J 1961 Phys. Rev. 1211195

[14] Hill D L and Wheeler J A 1953 Phys. Rev. 891102

[15] Swiatecki W J 1956 Phys. Rev. 101651

[16] Nix J R 1967 Ann. Phys. 4152

[17] Bohr A and Mottelson B R 1975 Nuclear Structure Vol. II (Benjamin Reading) p47

[18] Brack M, Ledergerber T and Pauli H C 1974 Nucl. Phys. A 234185

[19] Nilsson S G et al 1969 Nucl. Phys. A 1311

[20] Möller P et al 2009 Phys. Rev. C 79064304

[21] Möller P et al 2016 At. Data Nucl. Data Tables 109-110 1

[22] Lawrence J N P 1965 Phys. Rev. B 1391227

[23] Hasse R W 1969 Nucl. Phys. A 128609

[24] Hasse R W 1971 Ann. Phys. 68377

[25] Grammaticos B 1973 Phys. Lett. 443343

[26] Hasse R W 1977 Habilitationschrift (München)

[27] Swiatecki W J 1980 Prog. Part. Nucl. Phys. 4383

[28] Nix J R 1969 Nucl. Phys. A 130241

[29] Nix J R 1972 Ann. Rev. Nucl. Sc. 2265

[30] Blocki J and Swiatecki W J 1982 Berkeley Report LBL-12811

[31] Gherghescu R A 2003 Phys. Rev. C 67014309

[32] Pomorski et al 2018 Phys. Rev. C 97034319

[33] Dubray N 2008 Phys. Rev. C 77014310

[34] Diehl H and Greiner W 1974 Nucl. Phys. A 22929

[35] Gherghescu R A and Carjan N 2009 J. Phys. G: Nucl. Part. Phys. 36025106

[36] Royer G and Remaud B 1982 J. Phys. G: Nucl. Part. Phys. 8 L159

[37] Pashkevich V V 1971 Nucl. Phys. A 169275

[38] Mignen J, Royer G and Sebille F 1988 Nucl. Phys. A 489461

[39] Wong C Y 1973 Ann. Phys. 77279

[40] Chandrasekhar S 1969 Ellipsoidal figures of equilibrium (New Haven and London: Yale University Press)

[41] Schultheis H and Schultheis R 1975 J. Math. Phys. 16905

[42] Royer G and Haddad F 1995 J. Phys. G: Nucl. Part. Phys. 21339

[43] Quentin P 1969 J. Phys. (Paris) 30497

[44] Royer G, Piller C, Mignen J and Remaud B 1989 Nucl. Phys. A 494267

[45] Royer G and Piller C 1992 J. Phys. G: Nucl. Part. Phys. 181805

[46] Royer G, Gupta R K and Denisov V Yu 1998 Nucl. Phys. A 632275

[47] Viola V E and Seaborg G T 1966 J. Inorg. Nucl. Chem. 28741

[48] Poenaru D N, Ivascu M and Mazilu D 1982 Computer Physics Communications 25297

[49] Hourani E, Hussonnois M and Poenaru D N 1989 Ann. Phys. (Paris) 14311

[50] Poenaru D N and Greiner W 1991 Physica Scripta 44427

[51] Poenaru D N and Greiner W 1996 Handbook of Nuclear Properties (Oxford, Clarendon Press)

[52] Poenaru D N 1996 Nuclear Decay Modes (Institute of Physics Publishing, Bristol)

[53] Parkhomenko A and Sobiczewski A 2005 Acta Phys. Pol. B 361363

[54] Poenaru D N, Gherghescu R A and Greiner W 2005 Nucl.Phys. A 747182 
[55] Poenaru D N, Plonski I H, Gherghescu R A and Greiner W 2006 J. Phys. G: Nucl. Part. Phys. 321223

[56] Poenaru D N, Gherghescu R A and Carjan N 2007 Eur. Phys. Lett. 7762001

[57] Sobiczewski A and Pomorski K 2007 Prog. Part. Nucl. Phys. 58292

[58] Zhang H F and Royer G 2007 Phys. Rev. C 76047304

[59] Ni D D, Ren Z Z, Dong T K and Xu C 2008 Phys. Rev. C 78044310

[60] Poenaru D N 2010 Clusters in Nuclei Ed. C. Beck, Vol. 1, Lecture Notes in Physics 818 (Berlin: Springer) $\mathrm{p} 1$

[61] Poenaru D N, Gherghescu R A and Greiner W 2011 Phys. Rev. Lett. 107062503

[62] Poenaru D N, Gherghescu R A and Greiner W 2011 Phys. Rev. C 83014601

[63] Poenaru D N, Gherghescu R A and Greiner W 2012 Phys. Rev. C 85034615

[64] Poenaru D N, Gherghescu R A and Greiner W 2012 J. Phys. G: Nucl. Part. Phys. 39015105

[65] Bao X J et al 2012 J. Phys. G: Nucl. Part. Phys. 39095103

[66] Poenaru D N, Gherghescu R A and Greiner W 2013 J. Phys. G: Nucl. Part. Phys. 40105105

[67] Poenaru D N and Gherghescu R A 2014 J. Phys. G: Nucl. Part. Phys. 41125104

[68] Wang Y Z, Wang S J, Hou Z Y and Gu J Z 2015 Phys. Rev. C 92064301

[69] Denisov V Yu et al 2015 Phys. Rev. C 92014602

[70] Poenaru D N and Gherghescu R A 2016 Eur. Phys. J. A 52349

[71] Akrawy D T and Poenaru D N 2017 J. Phys. G: Nucl. Part. Phys. 44105105

[72] Poenaru D N and Gherghescu R A 2017 Eur. Phys. Lett. 11822001

[73] Zhang S et al 2017 Phys. Rev. C 95014311

[74] Akrawy D T et al 2018 Nucl. Phys. A 971130

[75] Poenaru D N, Stöcker H and Gherghescu R A 2018 Eur. Phys. J. A 5414

[76] Mazumbar I 2015 Pramana J. Phys. 85357

[77] Mignen J and Royer G 1987 J. Phys. G: Nucl. Part. Phys. 13987

[78] Royer G, Haddad F and Mignen J 1992 J. Phys. G: Nucl. Part. Phys. 182015

[79] Mignen J and Royer G 1990 J. Phys. G: Nucl. Part. Phys. 16 L227

[80] Lecolley J F et al 1994 Phys. Lett. B 325317

[81] Stone N T B et al 1997 Phys. Rev. Lett. 782084 